\section{PSA doubling time is not a surrogate for prostate-cancer- specific survival}

Several large, retrospective databases have shown that short prostate-specific-antigen doubling time (PSADT) can predict prostate-cancerspecific mortality. Pretreatment PSADT predicts treatment failure, while a high pretreatment PSA velocity is associated with an increased risk of death from prostate cancer following radiotherapy. Valicenti et al. used Prentice's Criteria of surrogacy to prospectively evaluate whether post-treatment PSADT could act as a surrogate end point for cause-specific survival (CSS) in men with locally advanced prostate cancer.

Patients were treated on Radiation Therapy Oncology Group (RTOG) Protocol 92-02, consisting of randomization to radiotherapy plus short-term ( $n=761)$ or long-term $(n=753)$ androgen deprivation. Follow-up was a median of 5.9 years. The long-term regimen was associated with a $43 \%$ reduction in prostate-cancer-specific mortality compared with the short-term regimen. Three of the four PSADT thresholds tested could be predicted by the randomized treatment and were predictive of CSS, as required by Prentice's Criteria. Although the fourth threshold, PSADT $<3$ months, predicted for a poor outcome, it did not meet the surrogacy requirements. Further analysis showed that the effect of PSADT on CSS was not independent of treatment.

PSADT does not, therefore, meet all of the criteria for surrogacy. Valicenti et al. recommend that clinical trials continue to use CSS or absolute survival as primary end points. An established surrogate for CSS would ideally, say the authors, be useful in determining a treatment strategy, as well as in extrapolating data and designing trials.

Original article Valicenti RK et al. (2006) Posttreatment prostatic-specific antigen doubling times as a surrogate endpoint for prostate cancer-specific survival: an analysis of Radiation Therapy Oncology Group Protocol 92-02. Int $J$ Radiat Oncol Biol Phys 66: 1064-1071

\section{The basis of receptor recognition by botulinum neurotoxin $B$}

Clostridium botulinum produces potent neurotoxins that block neurotransmitter release at femtomolar concentrations, and cause the neuroparalytic syndrome of botulism. So far, molecular details of toxin-cell recognition have been difficult to elucidate. Two new studies provide insight into receptor recognition by botulinum neurotoxin (BoNT) serotype B (BoNT/B), and describe potential therapeutic targets for vaccines or inhibitors.

The 'double receptor' hypothesis (proposed 20 years ago) posited that protein and ganglioside receptors on the neuronal plasma membrane interact with, and mediate entry of, BoNTs into nerve terminals. The only protein receptors for BoNT/B and BoNT serotype $G$ (BoNT/G) identified so far are synaptotagmins I and II (Sytl and Sytll). These synaptic-vesicle membrane proteins are vital for synaptic transmission in neuromuscular junctions. Both BoNT/B and BoNT/G form toxin-receptor complexes with Sytl and Sytll; these complexes are then taken up by neurons.

Until now, the protein receptor binding sites for BoNTs have been unknown. Jin et al. have elucidated the structure of the receptor-binding domain of BoNT/B in complex with the Sytll luminal domain: a helix is formed in the Sytll luminal domain that binds to a crevice on the distal tip of BoNT/B; Sytll and the ganglioside occupy two contiguous but nonoverlapping binding sites on BoNT/B adjacent to this crevice. To study the specificity of this interaction, the authors performed structure-based mutagenesis studies in vitro and in a neuronal assay. At or around the toxin-receptor interface, point mutations were introduced in BoNT/B or Sytll. Most of these mutations greatly reduced binding affinity. The significant attenuation of BoNT/B toxicity by single-site mutations at the BoNT/B-Sytll interface suggests that this interface could be a target for therapeutic agents that block the action of BoNT/B.

Although the protein receptors for BoNT/B and BoNT/G have been identified, the available information on recognition of cell-surface receptors is inconclusive. Chai et al. report crystallographic data that show the molecular details of toxin-receptor recognition of BoNT/B in complex with Sytll. Cocrystallization studies revealed that Sytll binds to a hydrophobic groove in the C-terminal subdomain of the BoNT/B carboxy-terminal domain. The short, helical, recognition domain of Sytll fits into this groove, and makes multiple hydrophobic contacts with BoNT/B at this interface, on formation of the complex. Structural analysis 